\title{
CONSERVAÇÃO DE SEMENTES DE AÇAÍ (Euterpe oleracea Mart.) ${ }^{1}$
}

\author{
WALNICE MARIA OLIVEIRA DO NASCIMENTO2; SILVIO MOURE CICERO; \\ ANA DIONÍSIA DA LUZ COELHO NOVEMBRE ${ }^{3}$
}

\begin{abstract}
RESUMO - Sementes de Euterpe oleracea são consideradas recalcitrantes e demandam ampliação do conhecimento sobre os fatores que interferem na sua conservação. Diante disso, o presente trabalho foi realizado com o objetivo de verificar os efeitos do teor de água da semente e da temperatura do ambiente na manutenção da qualidade das mesmas. Sementes da cultivar BRS Pará, com diferentes teores de água $(43,4 ; 37,4 ; 30,3 ; 26,1 ; 21,0 ; 15,1$ e $11,9 \%)$ e acondicionadas em sacos de polietileno foram armazenadas sob temperaturas de 20,15 e $10{ }^{\circ} \mathrm{C}$ durante 360 dias e submetidas a avaliações periódicas do teor de água, da germinação e do vigor. A secagem parcial até $37,4 \%$ de água não produz efeitos imediatos sobre a germinação e o vigor das sementes, a partir daí a secagem favorece, progressivamente, a deterioração das sementes e, ao atingirem 15,1\% as sementes não germinam. Após o armazenamento, sementes com $21,0 \%$ de água ou menos não germinam independentemente da temperatura. A associação de $43,4 \%$ de água na semente e o armazenamento em ambiente a $20{ }^{\circ} \mathrm{C}$ favorece a conservação das sementes por até 270 dias.
\end{abstract}

Termos para indexação: palmeira, secagem, deterioração, vigor.

\section{CONSERVATION OF Euterpe oleracea SEEDS}

\begin{abstract}
The açaí palm (Euterpe oleracea Mart.), grown principally for its palm heart and highly nutritious berrys, has recalcitrant seeds which cannot be stored for long periods because they do not resist drying or low temperatures. The effects of seed moisture content and storage temperature on the maintenance of seed quality were investigated using açaí palm cultivar BRS Pará seeds conditioned in polyethylene bags at moisture contents of $43.4 \%, 37.4 \%, 30.3 \%, 26.1 \%, 21.0 \%$, $15.1 \%$ and $11.9 \%$ and storage temperatures of $20{ }^{\circ} \mathrm{C}, 15{ }^{\circ} \mathrm{C}$ and $10{ }^{\circ} \mathrm{C}$ for 360 days, periodic samples being taken to determine moisture content, germination and vigor. At all the temperatures studied, partial drying to a water content of $37.4 \%$ did not produce any immediate effects on germination or vigor but as the water content dropped there was progressive deterioration until at $15.1 \%$ germination ceased. After 360 days, seeds with a water content of $21.0 \%$ or less did not germinate independent of storage temperature. Seeds with a water content of $43.4 \%$ stored at $20{ }^{\circ} \mathrm{C}$ remained viable for up to 270 days.
\end{abstract}

Index terms: açaí palm, drying, deterioration, vigor, water content.

${ }^{1}$ Submetido em 27/08/2009. Aceito para publicação em 08/12/2009.

Parte da Tese de Doutorado apresentada pelo primeiro autor à ESALQ/ USP. Piracicaba (SP).

${ }^{2}$ Embrapa Amazônia Oriental, Caixa Postal 48, CEP: 66095-100 Belém
(PA) E-mail: walnice@cpatu.embrapa.br.

${ }^{3}$ Departamento de Produção Vegetal, Escola Superior de Agricultura "Luiz de Queiroz", Caixa Postal 9, 13418-900 Piracicaba (SP). E-mail: smcicero@esalq.usp.br, adlcnove@esalq.usp.br 


\section{INTRODUÇÃO}

A palmeira Euterpe oleracea (Mart.), conhecida como açaizeiro tem se destacado economicamente pelo potencial mercadológico de seus produtos, representados, principalmente, pelo palmito e pelo suco extraído do fruto. Dessa maneira, a partir da disponibilidade de material propagativo, a exploração extrativista tem sido parcialmente substituída pela oriunda de campos de cultivo.

A estrutura utilizada para a propagação sexuada do açaizeiro, tecnologicamente denominada semente, que corresponde ao fruto desprovido de epicarpo e mesocarpo contém uma semente botânica, com eixo embrionário diminuto e abundante tecido endospermático, de formato esférico e representando $73 \%$ da massa do fruto completo (Villachica et al., 1996).

As sementes das espécies Euterpe oleracea Mart., Euterpe edulis Mart. (palmiteiro) e Euterpes espiritosantensis (palmito-vermelho) são admitidas como recalcitrantes (Carvalho et al., 1998; Araújo et al., 1994; Martins et al., 1999a) e, portanto, estão sujeitas à deterioração decorrente da secagem. Para sementes de açaí, o teor de água crítico esta na faixa de 34,2 a 37,4\%, abaixo do qual a viabilidade é reduzida (Martins et al., 1999b; Nascimento, 2006). Segundo Hong e Ellis (1996), as sementes recalcitrantes, além de incapazes de suportar adequadamente a dessecação abaixo de teores críticos de água, estão sujeitas ao rompimento de células, por expansão de volume, ao serem armazenadas sob temperatura de congelamento do conteúdo celular hidratado; adicionalmente, em espécies tropicais, essas sementes podem ser termicamente injuriadas em temperaturas entre 10 e 15 ${ }^{\circ} \mathrm{C}$. Essas exigências, para as condições de armazenamento contrapõem-se às indicadas para as sementes ortodoxas que, fundamentalmente, demandam baixos teores de água e de temperatura para a adequada manutenção da qualidade (Roberts, 1973).

De um modo geral, o armazenamento de sementes recalcitrantes deve proporcionar condições que previnam a perda de água, permitam o controle de microrganismos patogênicos e mantenham o suprimento adequado de oxigênio (King e Roberts, 1980). Para Bonner (1990), a embalagem empregada deve ser impermeável ao vapor de água e, ao mesmo tempo, permeável aos gases para possibilitar as trocas gasosas necessárias à manutenção da viabilidade.

Diversos estudos com espécies recalcitrantes comprovaram a importância na manutenção do teor de água nas sementes dessas espécies. Em experimento com sementes de pitomba (Talisia esculenta), armazenadas em ambiente sem controle de temperatura e umidade, foi verificada uma queda no teor de água das sementes de 40 para 24\%, 15 dias após o armazenamento reduzindo a emergência de plântulas de 88 para 16\% (Vieira e Gusmão, 2008).

Sementes de Euterpe edulis não germinam ao serem armazenadas com teores de água entre 14 e 18\% (Bovi e Cardoso, 1978). Entretanto, a germinação das sementes de palmiteiro não desidratadas, no interior da embalagem, constitui limitação em ambiente de armazenamento desprovido de controle térmico (Figliolia et al., 1987).

A conservação das sementes entre a colheita e a semeadura interfere na qualidade e na quantidade das plântulas obtidas e, em decorrência, no desempenho produtivo da população estabelecida no campo. O conhecimento disponível, particularmente a respeito da conservação de sementes de espécies nativas em fase de domesticação não é suficiente para embasar a definição de tecnologias de armazenamento.

Diante disso, o presente trabalho objetivou verificar os efeitos do teor de água da semente e da temperatura do ambiente na manutenção da qualidade fisiológica das sementes de Euterpe oleracea Mart.

\section{MATERIAL E MÉTODOS}

Foram utilizadas sementes de E. oleracea, da cultivar BRS Pará obtidas de frutos maduros (coloração do epicarpo roxo), os quais, imediatamente após a colheita foram submetidos à extração mecânica do epicarpo, à lavagem para eliminação dos resíduos e à seleção manual objetivando o descarte daqueles perfurados danificados por insetos.

Após a extração da polpa foi coletado o material constituinte do tratamento denominado $43,4 \%$ de água. Os demais tratamentos $(37,4 ; 30,3 ; 26,1 ; 21,0 ; 15,1$ e $11,9 \%$ de água) foram obtidos por meio do acompanhamento da perda de água das sementes, durante a secagem em equipamento com circulação forçada de $\operatorname{ar}\left(30 \pm 2{ }^{\circ} \mathrm{C}\right)$. Cada tratamento subsequente foi definido após a determinação do teor de água do tratamento anterior.

Durante a secagem as sementes foram distribuídas em camada única, sobre bandejas do secador. Para monitorar o processo, subamostras de sementes, com massa inicial conhecida foram acondicionadas em sacos de filó e distribuídas nas prateleiras do secador para pesagem a intervalos regulares. A massa final das amostras, correspondente aos teores de água desejados foi previamente determinada através da equação descrita por Cromarty et al. (1985): $\quad M_{f}=\frac{M_{i}\left(100-U_{i}\right)}{100-U_{f}}$, 
onde: $\mathrm{M}_{\mathrm{f}}=$ massa da amostra $(\mathrm{g})$ após a secagem; $\mathrm{M}_{\mathrm{i}}=$ massa da amostra $(\mathrm{g})$ antes da secagem; $\mathrm{U}_{\mathrm{i}}=$ teor de água $(\%)$ antes da secagem; $U_{\mathrm{f}}=$ grau de umidade (\%) desejado após a secagem.

À medida que foram sendo atingidos os teores de água desejados, as amostras foram homogeneizadas, divididas em 12 frações de 400 sementes cada, embaladas em sacos de polietileno transparente com dimensões de $30 \times 20 \mathrm{~cm}$, com $0,1 \mathrm{~mm}$ de espessura (lacrados à quente) e mantidas, provisoriamente, em ambiente a $10 \pm 1{ }^{\circ} \mathrm{C}$ até a obtenção dos demais tratamentos.

Uma vez obtidos os tratamentos relativos aos diferentes graus de umidade, as sementes foram armazenadas em câmaras com temperaturas controladas de $10 \pm 1 ; 15 \pm 1$ e $20 \pm 1{ }^{\circ} \mathrm{C}$.

No início do armazenamento e mensalmente, durante 360 dias, as sementes foram submetidas às avaliações discriminadas a seguir.

Determinação do teor de água: efetuada em estufa a $105 \pm 3{ }^{\circ} \mathrm{C}$, durante 24 horas, segundo as instruções das Regras para Análise de Sementes (Brasil, 1992), utilizandose quatro repetições de 10 sementes cada. Os resultados, expressos em porcentagem, foram calculados com base na massa úmida (bu).

Emergência de plântulas: realizada entre areia umedecida com $70 \%$ de sua capacidade de retenção de água (Brasil, 1992), empregando 50 sementes por repetição, semeadas a $1 \mathrm{~cm}$ de profundidade, em ambiente protegido e desprovido de controles de temperatura e de umidade relativa do ar. As sementes do interior das embalagens que se encontravam em início de germinação também foram utilizadas nos testes. Os resultados, obtidos aos 90 dias após a semeadura foram expressos em porcentagem de plântulas normais.

Índice de velocidade de emergência de plântulas: Foi obtida concomitantemente ao teste de germinação; para tanto, foram consideradas contagens, a cada três dias durante 90 dias, do número de plântulas emersas. Para o cálculo do índice de velocidade de emergência de plântulas (IVE), considerou a equação proposta por Maguire (1962):

$$
\operatorname{IVE}=\frac{E_{1}}{N_{1}}+\frac{E_{2}}{N_{2}}+\ldots+\frac{E_{n}}{N_{n}},
$$

onde: IVE $=$ índice de velocidade de emergência de plântula; $\mathrm{E}_{1}, \mathrm{E}_{2}, \ldots \mathrm{E}_{\mathrm{n}}=$ número de plântulas emersas nas primeira, segunda... e última contagens; $\mathrm{N}_{1}, \mathrm{~N}_{2}, \ldots, \mathrm{N}_{\mathrm{n}}=$ número de dias da semeadura à primeira, à segunda... e à última contagens.
Massa de matéria seca de plântulas: as plântulas normais separadas do restante da semente foram acondicionadas em sacos de papel e colocadas em estufa a $70 \pm 2{ }^{\circ} \mathrm{C}$ por 48 horas, previamente às pesagens. $\mathrm{A}$ massa de matéria seca ( $\mathrm{g} /$ plântula) foi obtida por meio do quociente entre o somatório de massa de plântulas normais e o número de sementes componentes da amostra.

Foi adotado o delineamento experimental inteiramente casualizado com quatro repetições, considerando-se sete tratamentos (teores de água), no início do armazenamento e 21 tratamentos (sete teores de água x três temperaturas de armazenamento) em cada época de avaliação. Para os dados obtidos durante o armazenamento, a análise de variância foi conduzida isoladamente em cada época de avaliação. Os dados em porcentagem foram transformados em arco seno da raiz quadrada de $x+\alpha / 100$ e os demais em raiz quadrada de $\mathrm{x}+\alpha$. As médias foram comparadas pelo teste de Tukey (5\% de probabilidade). Os dados do grau de umidade não foram submetidos à análise estatística.

\section{RESULTADOS E DISCUSSÃO}

\section{Antes do armazenamento}

Os diferentes teores de água obtidos para sementes de açaí ficaram próximos ao planejado para avaliação da qualidade fisiológica durante o período de armazenamento, indicando dessa maneira, a eficiência do método de secagem adotado para obtenção dos tratamentos (Tabela 1).

Os dados relativos às determinações de emergência, velocidade de emergência e massa de matéria seca de plântulas indicaram ausência de efeitos imediatos da secagem até $37,4 \%$ de água; a partir daí a deterioração foi proporcional à perda de água. Essa perda se tornou mais acentuada quando o teor de água foi reduzido para valores iguais ou inferiores a $26,1 \%$. A secagem progressiva intensificou o processo de deterioração das sementes, constatada pelas reduções da germinação e vigor. Quando a secagem atingiu o teor de água de $15,1 \%$ não houve emergência (Tabela 1). Os efeitos da secagem sobre o desempenho fisiológico das sementes de açaí foram também verificados por Nascimento e Silva (2005), com a anulação da germinação quando a desidratação atingiu $15,0 \%$ de água. $\mathrm{O}$ avanço no processo de secagem afetou o desempenho das sementes, de forma que ocorreu acentuada redução da emergência de plântulas e do vigor quando o teor de água foi inferior a 30,3\%, comprometendo assim a sua utilização (Tabela 1). 
TABELA 1. Teor de água de sementes, emergência, velocidade de emergência (IVE) e massa de matéria seca de plântulas (MS) de $E$. oleracea antes do armazenamento.

\begin{tabular}{cccc}
\hline Teor de água (\%) & Emergência (\%) & IVE & MS (g/plântula) \\
\hline 43,4 & $92 \mathrm{a}^{1}$ & $0,020 \mathrm{ab}$ & $0,440 \mathrm{a}$ \\
37,4 & $88 \mathrm{a}$ & $0,023 \mathrm{a}$ & $0,399 \mathrm{a}$ \\
30,3 & $80 \mathrm{~b}$ & $0,018 \mathrm{~b}$ & $0,272 \mathrm{~b}$ \\
26,1 & $42 \mathrm{c}$ & $0,016 \mathrm{~b}$ & $0,144 \mathrm{c}$ \\
21,0 & $07 \mathrm{~d}$ & $0,016 \mathrm{~b}$ & $0,021 \mathrm{~d}$ \\
15,1 & $00 \mathrm{e}$ & $0,000 \mathrm{c}$ & $0,000 \mathrm{e}$ \\
11,9 & $00 \mathrm{e}$ & $0,000 \mathrm{c}$ & $0,000 \mathrm{e}$ \\
\hline
\end{tabular}

${ }^{1}$ Nas colunas, médias seguidas pela mesma letra não diferem entre si (Tukey, 5\%).

\section{Durante o armazenamento}

A determinação do teor de água das sementes durante o período de armazenamento indicou a manutenção dos tratamentos relativos aos teores de água nas temperaturas consideradas. Entretanto, noúltimo mês do armazenamento, as sementes com $43,4 \%$ de água, que estavam sob as temperaturas de 15 e $20{ }^{\circ} \mathrm{C}$ tiveram aumento no teor de água em cerca de 7\% (Figura 1). Essa variação no teor de água das sementes armazenadas com alta umidade pode ser atribuída à elevada incidência de microrganismos ou a deterioração. Bilia et al. (1998) constataram variações no teor de água de sementes de Inga uruguensis, que foram atribuídas à deterioração das sementes ou ao aumento da incidência de microrganismos, os quais alteraram o microambiente. Alterações de $10 \%$ no teor de água em sementes de araucária, independentemente da embalagem e da temperatura utilizada para a conservação das sementes foram também identificadas por Piriz Carrilo et al. (2003).

A temperatura de armazenamento e os teores de água das sementes exerceram influência sobre a germinação e o vigor. Nos tratamentos em que as sementes foram armazenadas com 43,4 e 37,4\% de água houve diferenças significativas em relação aos demais tratamentos, com superioridade de desempenho germinativo; porém, quando comparados entre si, o tratamento contendo sementes com $43,4 \%$ de água foi superior na maioria das épocas de avaliadas. Entretanto, na condição de alta umidade, 90 dias após o armazenamento foi verificado que $20 \%$ das sementes germinaram no interior da embalagem, com acréscimo durante o período avaliado (Figura 2). Por outro lado, nas sementes com teor de água inferior a
$37,4 \%$ constatou-se redução significativa no desempenho fisiológico e, sementes com teor de água abaixo de $26,1 \%$ os dados foram, invariavelmente, nulos ou similares aos nulos (Tabela 2).

$\mathrm{O}$ armazenamento das sementes a $10 \quad{ }^{\circ} \mathrm{C}$ causou redução na germinação com diferenças entre os tratamentos, detectadas nos primeiros 30 dias. Sementes armazenadas com teores de água entre 43,4 e $26,1 \%$ perderam gradativamente a germinação até 90 dias de armazenamento, sendo que a partir daí a germinação foi anulada; sementes com teores de água abaixo de $26,1 \%$ proporcionaram valores nulos na germinação em todas as épocas estudadas (Tabela 2). Portanto, o armazenamento sob $10{ }^{\circ} \mathrm{C}$ foi o mais prejudicial para a manutenção da viabilidade das sementes de açaí. O efeito deletério causado por temperatura baixa também foi verificado em sementes de palmiteiro armazenadas a $5{ }^{\circ} \mathrm{C}$ (Andrade et al., 1996).

A secagem parcial das sementes até $37,4 \%$ de água propiciou condições de conservação na temperatura de $15{ }^{\circ} \mathrm{C}$ até 300 dias, sendo que a partir de 210 dias iniciouse a germinação das sementes no interior das embalagens (Figura 2). Entretanto, nessa temperatura verificou-se redução acentuada na germinação de sementes armazenadas com teores de água de 43,4 e 30,3\%. A temperatura de 15 ${ }^{\circ} \mathrm{C}$ inviabilizou a conservação das sementes de açaí com 43,4\% de água, a partir de 120 dias de armazenamento. Comportamento similar foi verificado para o armazenamento em ambiente a $10{ }^{\circ} \mathrm{C}$, para todos os tratamentos. Os efeitos negativos da temperatura sobre o desempenho fisiológico das sementes de açaí foram proporcionais à redução da temperatura. 

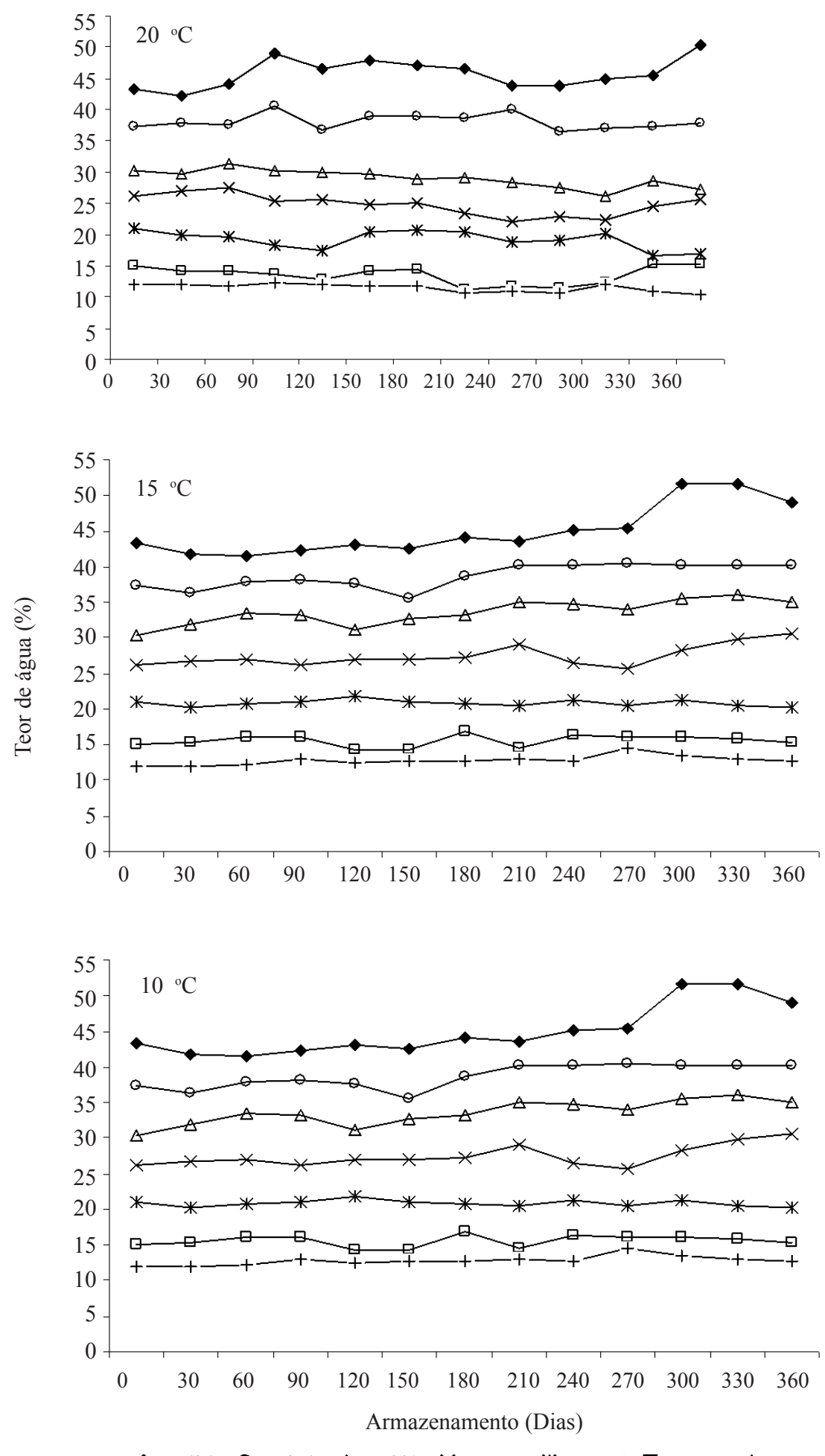

FIGURA 1. Teor de água de sementes de $E$. oleracea armazenadas em diferentes temperaturas. 

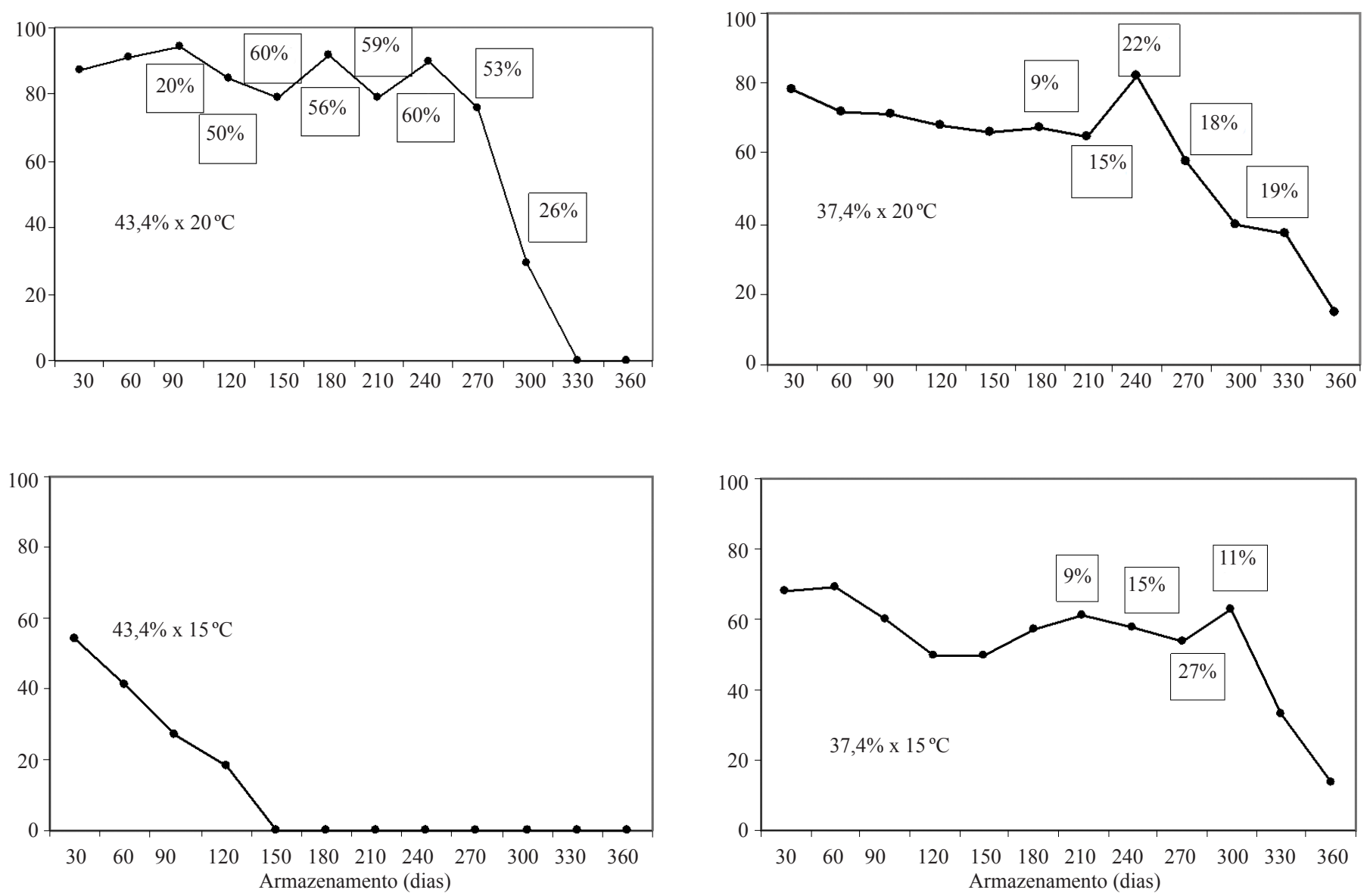

FIGURA 2. Emergência (\%) de plântulas de E. oleracea oriundas de sementes com 43,4 e 37,4\% de água, armazenadas a 20 e a $15{ }^{\circ} \mathrm{C}$. As porcentagens indicadas nos quadrículos correspondem às sementes que germinaram no interior da embalagem.

Durante o armazenamento em ambiente a $20{ }^{\circ} \mathrm{C}$ foi observado que sementes com 43,4 e $37,4 \%$ iniciaram a germinação no interior das embalagens, a partir de 90 e 180 dias, respectivamente. Diversos autores também verificaram a germinação de sementes recalcitrantes no interior da embalagem durante o período de armazenamento, a exemplo de Andrade (2001) quando armazenou sementes de $E$. edulis, a $15{ }^{\circ} \mathrm{C}$ com $44 \%$ de água e verificou que as mesmas iniciaram a germinação dentro da embalagem após 270 dias de armazenamento. Em sementes de três espécies de Shorea com $40 \%$ de água e armazenadas sob condições de $16{ }^{\circ} \mathrm{C}$, a germinação ocorreu no interior da embalagem (Tompsett, 1985). Em experimentos desenvolvidos por Corbineau e Côme (1986) foi verificado que todas as sementes de Symphonia globulifera (Clusiaceae) germinaram no interior da embalagem após 300 dias de armazenamento a $15{ }^{\circ} \mathrm{C}$.

A interpretação dos dados não identificou variação expressiva entre as indicações fornecidas pelos diferentes testes de vigor; desse modo, os tratamentos foram comparados segundo o desempenho predominante no conjunto das avaliações.

No armazenamento a $20{ }^{\circ} \mathrm{C}$ (Tabelas 3 e 4 ) houve diferença estatística entre os tratamentos, com desempenho superior das sementes com teores de água mais elevados (43,4 e 37,4\%) e, principalmente, das sementes com $43,4 \%$ de água. Os maiores valores para a velocidade de emergência de plântulas foram obtidos nas sementes armazenadas com 43,4\% de água, após 60 dias, a $20{ }^{\circ} \mathrm{C}$. Entretanto, no final do período de avaliação (330 e 360 dias), as sementes com 43,4\% de água, tiveram seu desempenho fisiológico anulado. Para as sementes com $30,3 \%$ e $26,1 \%$ de água, os dados foram similares aos nulos e abaixo de $26,1 \%$ foram invariavelmente nulos em todas as épocas de avaliação (Tabela 3). 
TABELA 2. Emergência (\%) de plântulas de E. oleracea oriundas de sementes: valores médios (\%) obtidos durante o armazenamento.

\begin{tabular}{|c|c|c|c|c|c|c|c|c|c|c|c|c|c|}
\hline \multirow{2}{*}{\multicolumn{2}{|c|}{$\begin{array}{c}\text { Tratamento } \\
\text { (temperatura/ } \\
\text { grau de umidade) }\end{array}$}} & \multicolumn{12}{|c|}{ Período de armazenamento (dia) } \\
\hline & & 30 & 60 & 90 & 120 & 150 & 180 & 210 & 240 & 270 & 300 & 330 & 360 \\
\hline \multirow{7}{*}{$20^{\circ} \mathrm{C} /$} & $43,4 \%$ & $87 a^{1}$ & $91 \mathrm{a}$ & $94 \mathrm{a}$ & $85 \mathrm{a}$ & $79 \mathrm{a}$ & $92 \mathrm{a}$ & $79 \mathrm{a}$ & $90 \mathrm{a}$ & $76 \mathrm{a}$ & $29 \mathrm{bc}$ & $00 \mathrm{c}$ & $00 \mathrm{~b}$ \\
\hline & $37,4 \%$ & $78 \mathrm{~b}$ & $72 a b$ & $71 \mathrm{~b}$ & $68 \mathrm{~b}$ & $66 a b$ & $67 \mathrm{~b}$ & $65 \mathrm{a}$ & $82 \mathrm{a}$ & $58 \mathrm{~b}$ & $40 \mathrm{~b}$ & $37 \mathrm{a}$ & $15 \mathrm{a}$ \\
\hline & $30,3 \%$ & $32 \mathrm{~h}$ & $30 \mathrm{e}$ & $22 \mathrm{f}$ & $00 \mathrm{f}$ & $08 \mathrm{~d}$ & $12 \mathrm{~d}$ & $00 \mathrm{c}$ & $12 \mathrm{c}$ & $00 \mathrm{e}$ & $00 \mathrm{~d}$ & $00 \mathrm{c}$ & $00 \mathrm{~b}$ \\
\hline & $26,1 \%$ & $23 \mathrm{i}$ & $25 \mathrm{e}$ & $11 \mathrm{~g}$ & $00 \mathrm{f}$ & $00 \mathrm{e}$ & $00 \mathrm{e}$ & $00 \mathrm{c}$ & $00 \mathrm{e}$ & $00 \mathrm{e}$ & $00 \mathrm{~d}$ & $00 \mathrm{c}$ & $00 \mathrm{~b}$ \\
\hline & $21,0 \%$ & $00 \mathrm{j}$ & $00 \mathrm{f}$ & $00 \mathrm{i}$ & $00 \mathrm{f}$ & $00 \mathrm{e}$ & $00 \mathrm{e}$ & $00 \mathrm{c}$ & $00 \mathrm{e}$ & $00 \mathrm{e}$ & $00 \mathrm{~d}$ & $00 \mathrm{c}$ & $00 \mathrm{~b}$ \\
\hline & $15,1 \%$ & $00 \mathrm{j}$ & $00 \mathrm{f}$ & $00 \mathrm{i}$ & $00 \mathrm{f}$ & $00 \mathrm{e}$ & $00 \mathrm{e}$ & $00 \mathrm{c}$ & $00 \mathrm{e}$ & $00 \mathrm{e}$ & $00 \mathrm{~d}$ & $00 \mathrm{c}$ & $00 \mathrm{~b}$ \\
\hline & $11,9 \%$ & $00 \mathrm{j}$ & $00 \mathrm{f}$ & $00 \mathrm{i}$ & $00 \mathrm{f}$ & $00 \mathrm{e}$ & $00 \mathrm{e}$ & $00 \mathrm{c}$ & $00 \mathrm{e}$ & $00 \mathrm{e}$ & $00 \mathrm{~d}$ & $00 \mathrm{c}$ & $00 \mathrm{~b}$ \\
\hline \multirow{7}{*}{$15^{\circ} \mathrm{C} /$} & $43,4 \%$ & $54 \mathrm{e}$ & $41 \mathrm{cde}$ & $27 \mathrm{f}$ & $18 \mathrm{e}$ & $00 \mathrm{e}$ & $00 \mathrm{e}$ & $00 \mathrm{c}$ & $00 \mathrm{e}$ & $00 \mathrm{e}$ & $00 \mathrm{~d}$ & $00 \mathrm{c}$ & $00 \mathrm{~b}$ \\
\hline & $37,4 \%$ & $68 \mathrm{c}$ & $69 \mathrm{ab}$ & $60 \mathrm{c}$ & $50 \mathrm{c}$ & $50 \mathrm{~b}$ & $57 \mathrm{~b}$ & $61 \mathrm{a}$ & $58 \mathrm{~b}$ & $54 \mathrm{c}$ & $63 \mathrm{a}$ & $33 \mathrm{~b}$ & $14 \mathrm{a}$ \\
\hline & $30,3 \%$ & $58 \mathrm{de}$ & $55 \mathrm{bcd}$ & $49 \mathrm{~d}$ & $36 \mathrm{~d}$ & $11 \mathrm{c}$ & $21 \mathrm{c}$ & $22 \mathrm{~b}$ & $06 \mathrm{~d}$ & $06 \mathrm{~d}$ & $22 \mathrm{c}$ & $00 \mathrm{c}$ & $00 \mathrm{~b}$ \\
\hline & $26,1 \%$ & $39 \mathrm{fg}$ & $34 \mathrm{de}$ & $12 \mathrm{~g}$ & $00 \mathrm{f}$ & $00 \mathrm{e}$ & $00 \mathrm{e}$ & $00 \mathrm{c}$ & $00 \mathrm{e}$ & $00 \mathrm{e}$ & $00 \mathrm{~d}$ & $00 \mathrm{c}$ & $00 \mathrm{~b}$ \\
\hline & $21,0 \%$ & $00 \mathrm{j}$ & $00 \mathrm{f}$ & $00 \mathrm{i}$ & $00 \mathrm{f}$ & $00 \mathrm{e}$ & $00 \mathrm{e}$ & $00 \mathrm{c}$ & $00 \mathrm{e}$ & $00 \mathrm{e}$ & $00 \mathrm{~d}$ & $00 \mathrm{c}$ & $00 \mathrm{~b}$ \\
\hline & $15,1 \%$ & $00 \mathrm{j}$ & $00 \mathrm{f}$ & $00 \mathrm{i}$ & $00 \mathrm{f}$ & $00 \mathrm{e}$ & $00 \mathrm{e}$ & $00 \mathrm{c}$ & $00 \mathrm{e}$ & $00 \mathrm{e}$ & $00 \mathrm{~d}$ & $00 \mathrm{c}$ & $00 \mathrm{~b}$ \\
\hline & $11,9 \%$ & $00 \mathrm{j}$ & $00 \mathrm{f}$ & $00 \mathrm{i}$ & $00 \mathrm{f}$ & $00 \mathrm{e}$ & $00 \mathrm{e}$ & $00 \mathrm{c}$ & $00 \mathrm{e}$ & $00 \mathrm{e}$ & $00 \mathrm{~d}$ & $00 \mathrm{c}$ & $00 \mathrm{~b}$ \\
\hline \multirow{7}{*}{$10^{\circ} \mathrm{C} /$} & $43,4 \%$ & $45 \mathrm{f}$ & $32 \mathrm{de}$ & $10 \mathrm{gh}$ & $00 \mathrm{f}$ & $00 \mathrm{e}$ & $00 \mathrm{e}$ & $00 \mathrm{c}$ & $00 \mathrm{e}$ & $00 \mathrm{e}$ & $00 \mathrm{~d}$ & $00 \mathrm{c}$ & $00 \mathrm{~b}$ \\
\hline & $37,4 \%$ & $39 \mathrm{fg}$ & $12 \mathrm{f}$ & $11 \mathrm{~g}$ & $00 \mathrm{f}$ & $10 \mathrm{~cd}$ & $10 \mathrm{~d}$ & $00 \mathrm{c}$ & $00 \mathrm{e}$ & $00 \mathrm{e}$ & $00 \mathrm{~d}$ & $00 \mathrm{c}$ & $00 \mathrm{~b}$ \\
\hline & $30,3 \%$ & $62 \mathrm{~cd}$ & $63 \mathrm{abc}$ & $38 \mathrm{e}$ & $00 \mathrm{f}$ & $00 \mathrm{e}$ & $00 \mathrm{~d}$ & $00 \mathrm{c}$ & $00 \mathrm{e}$ & $00 \mathrm{e}$ & $00 \mathrm{~d}$ & $00 \mathrm{c}$ & $00 \mathrm{~b}$ \\
\hline & $26,1 \%$ & $36 \mathrm{gh}$ & $32 \mathrm{de}$ & $06 \mathrm{~h}$ & $00 \mathrm{f}$ & $00 \mathrm{e}$ & $00 \mathrm{e}$ & $00 \mathrm{c}$ & $00 \mathrm{e}$ & $00 \mathrm{e}$ & $00 \mathrm{~d}$ & $00 \mathrm{c}$ & $00 \mathrm{~b}$ \\
\hline & $21,0 \%$ & $00 \mathrm{j}$ & $00 \mathrm{f}$ & $00 \mathrm{i}$ & $00 \mathrm{f}$ & $00 \mathrm{e}$ & $00 \mathrm{e}$ & $00 \mathrm{c}$ & $00 \mathrm{e}$ & $00 \mathrm{e}$ & $00 \mathrm{~d}$ & $00 \mathrm{c}$ & $00 \mathrm{~b}$ \\
\hline & $15,1 \%$ & $00 \mathrm{j}$ & $00 \mathrm{f}$ & $00 \mathrm{i}$ & $00 \mathrm{f}$ & $00 \mathrm{e}$ & $00 \mathrm{e}$ & $00 \mathrm{c}$ & $00 \mathrm{e}$ & $00 \mathrm{e}$ & $00 \mathrm{~d}$ & $00 \mathrm{c}$ & $00 \mathrm{~b}$ \\
\hline & $11,9 \%$ & $00 \mathrm{j}$ & $00 \mathrm{f}$ & $00 \mathrm{i}$ & $00 \mathrm{f}$ & $00 \mathrm{e}$ & $00 \mathrm{e}$ & $00 \mathrm{c}$ & $00 \mathrm{e}$ & $00 \mathrm{e}$ & $00 \mathrm{~d}$ & $00 \mathrm{c}$ & $00 \mathrm{~b}$ \\
\hline
\end{tabular}

${ }^{1}$ Médias seguidas pela mesma letra, nas colunas, não diferem entre si pelo teste de Tukey a 5\% de probabilidade.

Os resultados obtidos em sementes de açaí armazenadas nas temperaturas de 15 e $10{ }^{\circ} \mathrm{C}$ evidenciaram o rápido declínio no vigor das sementes. Com exceção dos tratamentos compreendidos entre 43,4 e $26,1 \%$ de água, até 120 dias para o ambiente a $15{ }^{\circ} \mathrm{C}$ e até 90 dias para o ambiente a $10{ }^{\circ} \mathrm{C}$, nos demais os valores foram nulos em todas as características avaliadas. Entretanto, é importante destacar que para as sementes armazenadas à $15^{\circ} \mathrm{C}$ com teores de água de $37,4 \%$ o vigor foi relativamente alto até 300 dias de armazenamento (Tabelas 3 e 4).

Em geral, as sementes recalcitrantes são intolerantes à redução da temperatura; de acordo com a maioria dos trabalhos publicados, valores inferiores a $15{ }^{\circ} \mathrm{C}$ reduzem a longevidade. Portanto, ainda não existem métodos satisfatórios para o armazenamento de sementes dessas espécies por períodos longos. Contudo, alguns pesquisadores consideram que é possível conservar, por curtos períodos e em temperaturas entre 2 e $20{ }^{\circ} \mathrm{C}$, sementes úmidas de espécies tropicais tais como o paubreu (Symphonia globulifera), a mangaba (Hancornia speciosa) e o camu-camu (Myrciaria dubia) (Corbineau e Côme, 1986; Oliveira e Valio, 1992; Gentil et al., 2004). Entretanto, não foi possível conservar satisfatoriamente as sementes de açaí com 43,4 e 37,4\% de água, em temperatura abaixo de $15{ }^{\circ} \mathrm{C}$, mesmo por um período de 30 dias (Tabelas 2, 3 e 4). 
Para a maioria das variáveis analisadas, durante o período de armazenamento, os valores de germinação e vigor indicaram como melhor tratamento, a associação de sementes com $43,4 \%$ de água e ambiente a $20{ }^{\circ} \mathrm{C}$. Por outro lado, os resultados da pesquisa indicaram que a desidratação parcial das sementes até $37,4 \%$ de água e o armazenamento sob temperatura de $20{ }^{\circ} \mathrm{C}$ conservam satisfatoriamente as sementes por até 240 dias, ampliando o período de armazenamento para as sementes de açaí, anteriormente relatados na literatura.

TABELA 3.Velocidade de emergência de plântulas de E. oleracea: valores médios obtidos durante o armazenamento.

\begin{tabular}{|c|c|c|c|c|c|c|c|c|c|c|c|c|c|}
\hline \multirow{2}{*}{\multicolumn{2}{|c|}{$\begin{array}{c}\text { Tratamento } \\
\text { (temperatura/ } \\
\text { grau de } \\
\text { umidade) }\end{array}$}} & \multicolumn{12}{|c|}{ Período de armazenamento (dia) } \\
\hline & & 30 & 60 & 90 & 120 & 150 & 180 & 210 & 240 & 270 & 300 & 330 & 360 \\
\hline \multirow{7}{*}{$20^{\circ} \mathrm{C} /$} & $43,4 \%$ & $0,033 \mathrm{a}^{1}$ & $0,103 \mathrm{a}$ & $0,293 \mathrm{a}$ & $0,242 \mathrm{a}$ & $0,276 \mathrm{a}$ & $0,306 \mathrm{a}$ & $0,319 \mathrm{a}$ & $0,323 \mathrm{a}$ & $0,308 \mathrm{a}$ & $0,301 \mathrm{a}$ & $0,000 \mathrm{~b}$ & $0,000 \mathrm{~b}$ \\
\hline & $37,4 \%$ & $0,021 \mathrm{c}$ & $0,025 \mathrm{~b}$ & $0,026 \mathrm{~b}$ & $0,046 \mathrm{~b}$ & $0,069 \mathrm{~b}$ & $0,115 \mathrm{~b}$ & $0,056 \mathrm{~b}$ & $0,095 \mathrm{~b}$ & $0,112 b$ & $0,159 \mathrm{c}$ & $0,146 \mathrm{a}$ & 0,015 a \\
\hline & $30,3 \%$ & $0,013 \mathrm{f}$ & $0,013 \mathrm{~d}$ & 0,013 de & $0,000 \mathrm{c}$ & $0,013 \mathrm{c}$ & $0,000 \mathrm{~d}$ & $0,000 \mathrm{~d}$ & $0,012 \mathrm{~d}$ & $0,000 \mathrm{e}$ & $0,000 \mathrm{~d}$ & $0,000 \mathrm{~b}$ & $0,000 \mathrm{~b}$ \\
\hline & $26,1 \%$ & $0,013 \mathrm{f}$ & $0,012 \mathrm{~d}$ & $0,012 \mathrm{de}$ & $0,000 \mathrm{c}$ & $0,000 \mathrm{c}$ & $0,000 \mathrm{~d}$ & $0,000 \mathrm{~d}$ & $0,000 \mathrm{e}$ & $0,000 \mathrm{e}$ & $0,000 \mathrm{~d}$ & $0,000 \mathrm{~b}$ & $0,000 \mathrm{~b}$ \\
\hline & $21,0 \%$ & $0,000 \mathrm{~g}$ & $0,000 \mathrm{e}$ & $0,000 \mathrm{f}$ & $0,000 \mathrm{c}$ & $0,000 \mathrm{c}$ & $0,000 \mathrm{~d}$ & $0,000 \mathrm{~d}$ & $0,000 \mathrm{e}$ & $0,000 \mathrm{e}$ & $0,000 \mathrm{~d}$ & $0,000 \mathrm{~b}$ & $0,000 \mathrm{~b}$ \\
\hline & $15,1 \%$ & $0,000 \mathrm{~g}$ & $0,000 \mathrm{e}$ & $0,000 \mathrm{f}$ & $0,000 \mathrm{c}$ & $0,000 \mathrm{c}$ & $0,000 \mathrm{~d}$ & $0,000 \mathrm{~d}$ & $0,000 \mathrm{e}$ & $0,000 \mathrm{e}$ & $0,000 \mathrm{~d}$ & $0,000 \mathrm{~b}$ & $0,000 \mathrm{~b}$ \\
\hline & $11,9 \%$ & $0,000 \mathrm{~g}$ & $0,000 \mathrm{e}$ & $0,000 \mathrm{f}$ & $0,000 \mathrm{c}$ & $0,000 \mathrm{c}$ & $0,000 \mathrm{~d}$ & $0,000 \mathrm{~d}$ & $0,000 \mathrm{e}$ & $0,000 \mathrm{e}$ & $0,000 \mathrm{~d}$ & $0,000 \mathrm{~b}$ & $0,000 \mathrm{~b}$ \\
\hline \multirow{7}{*}{$15^{\circ} \mathrm{C} /$} & $43,4 \%$ & $0,024 \mathrm{bc}$ & $0,017 \mathrm{~cd}$ & $0,018 \mathrm{c}$ & $0,014 \mathrm{c}$ & $0,000 \mathrm{c}$ & $0,000 \mathrm{~d}$ & $0,000 \mathrm{~d}$ & $0,000 \mathrm{e}$ & $0,000 \mathrm{e}$ & $0,000 \mathrm{~d}$ & $0,000 \mathrm{~b}$ & $0,000 \mathrm{~b}$ \\
\hline & $37,4 \%$ & $0,023 \mathrm{bc}$ & $0,020 \mathrm{bc}$ & $0,017 \mathrm{~cd}$ & $0,014 \mathrm{c}$ & $0,015 \mathrm{c}$ & $0,041 \mathrm{c}$ & $0,053 \mathrm{c}$ & $0,021 \mathrm{c}$ & $0,044 \mathrm{c}$ & $0,183 \mathrm{~b}$ & $0,016 \mathrm{~b}$ & $0,011 \mathrm{ab}$ \\
\hline & $30,3 \%$ & $0,018 \mathrm{de}$ & $0,015 \mathrm{~cd}$ & $0,016 \mathrm{~cd}$ & $0,013 \mathrm{c}$ & $0,013 \mathrm{c}$ & $0,013 \mathrm{~d}$ & $0,013 \mathrm{~cd}$ & $0,013 \mathrm{~d}$ & $0,013 \mathrm{~d}$ & $0,013 \mathrm{~d}$ & $0,000 \mathrm{~b}$ & $0,000 \mathrm{~b}$ \\
\hline & $26,1 \%$ & $0,014 \mathrm{f}$ & $0,013 \mathrm{~d}$ & $0,012 \mathrm{de}$ & $0,000 \mathrm{c}$ & $0,000 \mathrm{c}$ & $0,000 \mathrm{~d}$ & $0,000 \mathrm{~d}$ & $0,000 \mathrm{e}$ & $0,000 \mathrm{e}$ & $0,000 \mathrm{~d}$ & $0,000 \mathrm{~b}$ & $0,000 \mathrm{~b}$ \\
\hline & $21,0 \%$ & $0,000 \mathrm{~g}$ & $0,000 \mathrm{e}$ & $0,000 \mathrm{f}$ & $0,000 \mathrm{c}$ & $0,000 \mathrm{c}$ & $0,000 \mathrm{~d}$ & $0,000 \mathrm{~d}$ & $0,000 \mathrm{e}$ & $0,000 \mathrm{e}$ & $0,000 \mathrm{~d}$ & $0,000 \mathrm{~b}$ & $0,000 \mathrm{~b}$ \\
\hline & $15,1 \%$ & $0,000 \mathrm{~g}$ & $0,000 \mathrm{e}$ & $0,000 \mathrm{f}$ & $0,000 \mathrm{c}$ & $0,000 \mathrm{c}$ & $0,000 \mathrm{~d}$ & $0,000 \mathrm{~d}$ & $0,000 \mathrm{e}$ & $0,000 \mathrm{e}$ & $0,000 \mathrm{~d}$ & $0,000 \mathrm{~b}$ & $0,000 \mathrm{~b}$ \\
\hline & $11,9 \%$ & $0,000 \mathrm{e}$ & $0,000 \mathrm{e}$ & $0,000 \mathrm{f}$ & $0,000 \mathrm{c}$ & $0,000 \mathrm{c}$ & $0,000 \mathrm{~d}$ & $0,000 \mathrm{~d}$ & $0,000 \mathrm{e}$ & $0,000 \mathrm{e}$ & $0,000 \mathrm{~d}$ & $0,000 \mathrm{~b}$ & $0,000 \mathrm{~b}$ \\
\hline \multirow{7}{*}{$10^{\circ} \mathrm{C} /$} & $43,4 \%$ & $0,025 \mathrm{~b}$ & $0,018 \mathrm{~cd}$ & $0,016 \mathrm{~cd}$ & $0,000 \mathrm{c}$ & $0,000 \mathrm{c}$ & $0,000 \mathrm{~d}$ & $0,000 \mathrm{~d}$ & $0,000 \mathrm{e}$ & $0,000 \mathrm{e}$ & $0,000 \mathrm{~d}$ & $0,000 \mathrm{~b}$ & $0,000 \mathrm{~b}$ \\
\hline & $37,4 \%$ & $0,018 \mathrm{e}$ & $0,017 \mathrm{~cd}$ & $0,012 \mathrm{de}$ & $0,000 \mathrm{c}$ & $0,012 \mathrm{c}$ & $0,012 \mathrm{~d}$ & $0,000 \mathrm{~d}$ & $0,000 \mathrm{e}$ & $0,000 \mathrm{e}$ & $0,000 \mathrm{~d}$ & $0,000 \mathrm{~b}$ & $0,000 \mathrm{~b}$ \\
\hline & $30,3 \%$ & $0,021 \mathrm{~cd}$ & $0,015 \mathrm{~cd}$ & $0,012 \mathrm{de}$ & $0,000 \mathrm{c}$ & $0,000 \mathrm{c}$ & $0,000 \mathrm{~d}$ & $0,000 \mathrm{~d}$ & $0,000 \mathrm{e}$ & $0,000 \mathrm{e}$ & $0,000 \mathrm{~d}$ & $0,000 \mathrm{~b}$ & $0,000 \mathrm{~b}$ \\
\hline & $26,1 \%$ & 0,015 ef & $0,013 \mathrm{~d}$ & $0,012 \mathrm{de}$ & $0,000 \mathrm{c}$ & $0,000 \mathrm{c}$ & $0,000 \mathrm{~d}$ & $0,000 \mathrm{~d}$ & $0,000 \mathrm{e}$ & $0,000 \mathrm{e}$ & $0,000 \mathrm{~d}$ & $0,000 \mathrm{~b}$ & $0,000 \mathrm{~b}$ \\
\hline & $21,0 \%$ & $0,000 \mathrm{~g}$ & $0,000 \mathrm{e}$ & $0,000 \mathrm{f}$ & $0,000 \mathrm{c}$ & $0,000 \mathrm{c}$ & $0,000 \mathrm{~d}$ & $0,000 \mathrm{~d}$ & $0,000 \mathrm{e}$ & $0,000 \mathrm{e}$ & $0,000 \mathrm{~d}$ & $0,000 \mathrm{~b}$ & $0,000 \mathrm{~b}$ \\
\hline & $15,1 \%$ & $0,000 \mathrm{~g}$ & $0,000 \mathrm{e}$ & $0,000 \mathrm{f}$ & $0,000 \mathrm{c}$ & $0,000 \mathrm{c}$ & $0,000 \mathrm{~d}$ & $0,000 \mathrm{~d}$ & $0,000 \mathrm{e}$ & $0,000 \mathrm{e}$ & $0,000 \mathrm{~d}$ & $0,000 \mathrm{~b}$ & $0,000 \mathrm{~b}$ \\
\hline & $11,9 \%$ & $0,000 \mathrm{~g}$ & $0,000 \mathrm{e}$ & $0,000 \mathrm{f}$ & $0,000 \mathrm{c}$ & $0,000 \mathrm{c}$ & $0,000 \mathrm{~d}$ & $0,000 \mathrm{~d}$ & $0,000 \mathrm{e}$ & $0,000 \mathrm{e}$ & $0,000 \mathrm{~d}$ & $0,000 \mathrm{~b}$ & $0,000 \mathrm{~b}$ \\
\hline
\end{tabular}

${ }^{1}$ Médias seguidas pela mesma letra, nas colunas, não diferem entre si pelo teste de Tukey a $5 \%$ de probabilidade. 
TABELA 4. Massa da matéria seca de plântulas de E. oleracea: valores médios (g/plântula) obtidos durante o armazenamento das sementes.

\begin{tabular}{|c|c|c|c|c|c|c|c|c|c|c|c|c|c|}
\hline \multirow{2}{*}{\multicolumn{2}{|c|}{$\begin{array}{c}\text { Tratamento } \\
\text { (temperatura/ } \\
\text { grau de umidade) }\end{array}$}} & \multicolumn{12}{|c|}{ Período de armazenamento (dia) } \\
\hline & & 30 & 60 & 90 & 20 & 150 & 180 & 210 & 240 & 270 & 300 & 330 & 360 \\
\hline \multirow{7}{*}{$20^{\circ} \mathrm{C} /$} & $43,4 \%$ & $0,281 \mathrm{a}^{1}$ & 364 a & $0,320 \mathrm{a}$ & $227 \mathrm{a}$ & 0,216 a & $0,138 \mathrm{a}$ & $0,157 \mathrm{a}$ & $0,169 \mathrm{a}$ & $0,121 \mathrm{a}$ & $0,083 \mathrm{~b}$ & 0,000 &, $000 \mathrm{c}$ \\
\hline & 37 & & & & & $\mathrm{a}$ & $1 \mathrm{a}$ & $0,102 a b$ & $0,184 \mathrm{a}$ & $09 \mathrm{~b}$ & $0,076 \mathrm{~b}$ & ( & . \\
\hline & $30,3 \%$ & $70 \mathrm{~cd}$ & 43 ef & $0,023 \mathrm{~cd}$ & $000 \mathrm{~d}$ & $0,038 \mathrm{~b}$ & $0,000 \mathrm{c}$ & $0,000 \mathrm{~d}$ & $0,017 \mathrm{~b}$ & $0,000 \mathrm{c}$ & $0,000 \mathrm{~d}$ & 0,00 & . \\
\hline & $26,1 \%$ & $72 \mathrm{~cd}$ & $137 \mathrm{f}$ & $0,029 \mathrm{~cd}$ & $000 \mathrm{~d}$ & $0,000 \mathrm{~d}$ & $0,000 \mathrm{c}$ & $0,000 \mathrm{~d}$ & $0,000 \mathrm{e}$ & $0,000 \mathrm{c}$ & $0,000 \mathrm{~d}$ & 0,000 &, $000 \mathrm{c}$ \\
\hline & & $00 \mathrm{f}$ & $00 \mathrm{~g}$ & $000 \mathrm{e}$ & $0,000 \mathrm{~d}$ & $0,000 \mathrm{~d}$ &, $000 \mathrm{c}$ & $0,000 \mathrm{~d}$ & $0,000 \mathrm{e}$ & $0,000 \mathrm{c}$ & $0,000 \mathrm{~d}$ & 0,0 & $000 \mathrm{c}$ \\
\hline & & $0 \mathrm{f}$ & $\mathrm{g}$ & e & $0 \mathrm{~d}$ & $d$ & $00 \mathrm{c}$ & $00 \mathrm{~d}$ & $0,000 \mathrm{e}$ & & $0,000 \mathrm{~d}$ & 0,0 & $00 \mathrm{c}$ \\
\hline & & & & & d & 0 & c & & & & & & $\mathrm{c}$ \\
\hline \multirow{7}{*}{$15^{\circ} \mathrm{C} /$} & 43 & 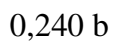 & $0,207 \mathrm{~b}$ & $0,153 \mathrm{bc}$ & $028 \mathrm{c}$ & $0,000 \mathrm{~d}$ & $0,000 \mathrm{c}$ & $0,000 \mathrm{~d}$ & $0,001 \mathrm{e}$ & $c$ & $0,000 \mathrm{~d}$ & 0,0 & $000 \mathrm{c}$ \\
\hline & $37,4 \%$ & $51 \mathrm{~b}$ & $194 \mathrm{~cd}$ & $0,175 \mathrm{bc}$ & $0,103 \mathrm{~b}$ & $0,099 \mathrm{~b}$ & $0,011 \mathrm{~b}$ & $0,054 \mathrm{bc}$ & $0,062 \mathrm{c}$ & $0,103 \mathrm{~b}$ & $0,114 \mathrm{a}$ & 0,0 & $013 \mathrm{~b}$ \\
\hline & $30,3 \%$ & $0,211 \mathrm{bc}$ & $0,155 \mathrm{ef}$ & $0,150 \mathrm{bc}$ & $0,068 \mathrm{bc}$ & $0,012 \mathrm{c}$ & $0,003 \mathrm{bc}$ & $0,010 \mathrm{c}$ & $0,005 \mathrm{~d}$ & $0,009 \mathrm{c}$ & $0,025 \mathrm{c}$ & 0,00 & $0,000 \mathrm{c}$ \\
\hline & $26,1 \%$ & $0,182 \mathrm{de}$ & $0,137 \mathrm{f}$ & $0,029 \mathrm{~cd}$ & $0,000 \mathrm{~d}$ & $0,000 \mathrm{~d}$ & $0,000 \mathrm{c}$ & $0,000 \mathrm{~d}$ & $0,000 \mathrm{e}$ & $0,000 \mathrm{c}$ & $0,000 \mathrm{~d}$ & 0,00 &, $000 \mathrm{c}$ \\
\hline & & & & & & $0,000 \mathrm{~d}$ & $0,000 \mathrm{c}$ &, $000 \mathrm{a}$ & & & $0,000 \mathrm{~d}$ & 0,0 & $000 \mathrm{c}$ \\
\hline & & $0,000 \mathrm{f}$ & & & $0,000 \mathrm{~d}$ & $0,000 \mathrm{~d}$ & $0,000 \mathrm{c}$ & $0,000 \mathrm{~d}$ & $0,000 \mathrm{e}$ & & $0,000 \mathrm{~d}$ & 0,00 &, $000 \mathrm{c}$ \\
\hline & & & & & & & & & & & $0,000 \mathrm{~d}$ & &, 000 \\
\hline \multirow{7}{*}{$10^{\circ} \mathrm{C} /$} & & & & & $0,000 \mathrm{~d}$ & $0,000 \mathrm{~d}$ & $0,000 \mathrm{c}$ & $0,000 \mathrm{~d}$ & & & $0,000 \mathrm{~d}$ & &, 000 \\
\hline & $37,4 \%$ & $0,190 \mathrm{c}$ & $0,068 \mathrm{~g}$ & $0,015 \mathrm{~cd}$ & $0,000 \mathrm{~d}$ & $0,000 \mathrm{~d}$ & $0,000 \mathrm{c}$ & $0,000 \mathrm{~d}$ & $0,000 \mathrm{e}$ & $0,000 \mathrm{c}$ & $0,000 \mathrm{~d}$ & 0,000 &, $000 \mathrm{c}$ \\
\hline & $30,3 \%$ & $0,239 \mathrm{~b}$ & $0,151 \mathrm{ef}$ & $0,138 \mathrm{bc}$ & $0,000 \mathrm{~d}$ & $0,000 \mathrm{~d}$ & $0,000 \mathrm{c}$ & $0,000 \mathrm{~d}$ & $0,000 \mathrm{e}$ & $0,000 \mathrm{c}$ & $0,000 \mathrm{~d}$ & 0,000 &, $000 \mathrm{c}$ \\
\hline & $26,1 \%$ & & & $0,011 \mathrm{~cd}$ & $0,000 \mathrm{~d}$ & $0,000 \mathrm{~d}$ & $0,000 \mathrm{c}$ & $0,000 \mathrm{~d}$ & $0,000 \mathrm{e}$ & $0,000 \mathrm{c}$ & $0,000 \mathrm{~d}$ & 0,000 & $0,000 \mathrm{c}$ \\
\hline & $21,0 \%$ & $0,000 \mathrm{f}$ & $0,000 \mathrm{~g}$ & $0,000 \mathrm{e}$ & $0,000 \mathrm{~d}$ & $0,000 \mathrm{~d}$ & $0,000 \mathrm{c}$ & $0,000 \mathrm{~d}$ & $0,000 \mathrm{e}$ & $0,000 \mathrm{c}$ & $0,000 \mathrm{~d}$ & 0,000 & $0,000 \mathrm{c}$ \\
\hline & $15,1 \%$ & $0,000 \mathrm{f}$ & $0,000 \mathrm{~g}$ & $0,000 \mathrm{e}$ & $0,000 \mathrm{~d}$ & $0,000 \mathrm{~d}$ & $0,000 \mathrm{c}$ & $0,000 \mathrm{~d}$ & $0,000 \mathrm{e}$ & $0,000 \mathrm{c}$ & $0,000 \mathrm{~d}$ & 0,00 & $0,000 \mathrm{c}$ \\
\hline & $11,9 \%$ & $0,000 \mathrm{f}$ & $0,000 \mathrm{~g}$ & $0,000 \mathrm{e}$ & $0,000 \mathrm{~d}$ & $0,000 \mathrm{~d}$ & $0,000 \mathrm{c}$ & $0,000 \mathrm{~d}$ & $0,000 \mathrm{e}$ & $0,000 \mathrm{c}$ & $0,000 \mathrm{~d}$ & $0,000 \mathrm{c}$ & $0,000 \mathrm{c}$ \\
\hline
\end{tabular}

${ }^{1}$ Médias seguidas pela mesma letra, nas colunas, não diferem entre si pelo teste de Tukey a $5 \%$ de probabilidade.

\section{CONCLUSÕES}

Independentemente da temperatura de armazenamento, nas sementes de E. oleracea com $21,0 \%$ de água não ocorre emergência de plântula.

A conservação de sementes de E. oleracea com teor de água de $37,4 \%$ em ambiente com temperatura constante de $20{ }^{\circ} \mathrm{C}$ ocorre por até 270 dias.

\section{AGRADECIMENTO}

Os autores agradecem ao Prof. Walter Rodrigues da Silva, in memorian, pela orientação recebida durante a realização da pesquisa.

\section{REFERÊNCIAS}

ANDRADE, A.C.S. The effect of moisture content and temperature on the longevity of heart of palm seeds (Euterpe edulis). Seed Science and Technology, v.29, n.1, p.171-182, 2001.

ANDRADE, A.C.S.; MALAVASI, M.M.; COSTA, F.A. Conservação de palmiteiro (Euterpe edulis Mart.): efeito da temperatura de armazenamento e do grau de umidade das sementes. Revista Brasileira de Sementes, v.18, n.2, p.149$155,1996$.

ARAÚJO, E.F.; SILVA, R.F.; ARAÚJO, R.F. Avaliação da qualidade de sementes de açaí armazenadas em diferentes embalagens. Revista Brasileira de Sementes, v.1, n.16, p.76-79, 1994. 
BILIA, D.A.C.; MARCOS FILHO. J.; NOVEMBRE, A.D.L.C. Conservação da qualidade fisiológica de sementes de Inga uruguensis Hook. Et Arn. Revista Brasileira de Sementes, v.20, n.1, p.48-54, 1998.

BONNER, F.T. Storage of seeds: potential and limitations for germoplasm conservation. Forest Ecology and Management, v.35, n.1, p.35-43, 1990.

BOVI, M.L.A.; CARDOSO, M. Conservação de sementes de palmiteiro (Euterpe edulis Mart.). Bragantia, v.37, n.1, p.65-71, 1978.

CARVALHO, J.E.U.; NASCIMENTO, W.M.O.; MÜLLER, C.H. Características físicas e de germinação de sementes de espécies frutíferas nativas da Amazônia. Belém: Embrapa, 1998. 18p. (Boletim de Pesquisa, 203).

CORBINEAU, F.; CÔME, D. Storage of recalcitrant seeds of four tropical species. Seed Science and Technology, v.16, n.1, p.97-103, 1986.

CROMARTY, A.S.; ELLIS, R.H.; ROBERTS, E.H. Design of seed storage facilities for genetic conservation. Rome: IPGRI, 1985. 100p.

FIGLIOLIA, M.B.; SILVA, A.; YAMAZOE, G.; SIQUEIRA, A.C.M.F. Conservação de sementes de Euterpe edulis Mart. em diferentes embalagens e ambiente de armazenamento. Boletim Técnico do Instituto Florestal, v.41, n.2, p.355368, 1987.

GENTIL，D.F.O.; SILVA, W.R.; FERREIRA, S.A.N. Conservação de sementes de Myrciaria dubia (H.B.K.) McVaugh. Bragantia, v.63, n.3, p.421-430, 2004.

HONG, T.D.; ELLIS, R.H. A protocol to determine seed storage behavior. Rome: IPGRI, 1996. 62p. (Technical Bulletin, 1).

KING, M.W.; ROBERTS, E.H. A strategy for future research into the storage of recalcitrant seeds. In: CHIN, H.F.; ROBERTS, E.H. (Ed.) Recalcitrant crop seeds. Kuala Lumpur: Tropical Press, 1980. cap.5, p.90-110.

MAGUIRE, J.D. Speed of germination: aid in selection and evaluation for seedling emergence and vigour. Crop Science, v.2, n.2, p.176-177, 1962.
MARTINS, C.C.; NAKAGAWA, J.; BOVI, M.L.A. Tolerância à dessecação de sementes de palmito-vermelho (Euterpe espiritosantensis Fernandes). Revista Brasileira de Botânica, v.22, n.3, p.391-396, 1999a.

MARTINS, C.C.; NAKAGAWA, J.; BOVI, M.L.A.; STANGUERLIM, H. Teores de água crítico e letal para sementes de açaí (Euterpe oleracea Mart. - Palmae). Revista Brasileira de Sementes, v. 21, n.1, p.125-132, 1999 b.

NASCIMENTO, W.M.O; SILVA, W.R. Comportamento fisiológico de sementes de açaí (Euterpe oleracea Mart.) submetidas à desidratação. Revista Brasileira de Fruticultura, v. 27, n.3, p.349-351, 2005.

NASCIMENTO, W. M. O. Conservação de sementes de açaí (Euterpe oleracea Mart.). 2006. 60 f. Tese (Doutorado em Agronomia/Fitotecnia) - Escola Superior de Agricultura Luiz de Queiroz, Universidade de São Paulo, Piracicaba, 2006.

OLIVEIRA, L.M.; VALIO, I. F.M. Effects of moisture content on germination of seeds of Hancornia speciosa Gom. (Apocynaceae) Annals of Botany, v.69, n.1, p.1-5, 1992.

PIRIZ CARILLO, V.; CHAVES, A.; FASSOLA, H.; MUGRIDGE, A. A refrigerated storage of seeds of Araucaria angustifólia (Bert.) O. Kuntze over a period of 24 months. Seed Science and Technology, v.31, n.2, p.411-429, 2003.

ROBERTS, E.H. Predicting the storage life of seeds. Seed Science and Technology, v.1, n.3, p.499-514, 1973.

TOMPSETT, P.B. The influence of moisture content and temperature on the viability of Shorea almon, Shorea robusta and Shorea roxburghii seed. Canadian Journal of Forest Research, v.15, n.1, p.107-116, 1985.

VIEIRA, F. de A.; GUSMÃO, E. Biometria, armazenamento e emergência de plântulas de Talisia esculenta Radlk. (Sapindaceae). Ciência Agrotécnica, v.32, n.4, p.10731079, 2008.

VILLACHICA, H.; CARVALHO, J.E.U.; MÜLLER, C.H.; DIAZ S.C.; ALMANZA, M. Frutales y hortalizas promisorios de la Amazonia. Lima: Tratado de Cooperación Amazónica, 1996. p.33-42 (TCA-SPT,44). 